Heft 23, p. 86 (1905).-Matsumura, Ind. Pl. Jap. II-2, p. 416 (1912).Mryabe et Kudo, Mater. Fl. Hokk. VIII, p. 32 (1918).-Komarov et Klob.Alisova, Key Pl. Far East. Reg. USSR. II, p. 780, t. 238, 1-4 (1932).

Syn. Myriophyllum verticillatum $\beta$. ussuriense REGEL, Tent. Fl. Ussur p. 60, tab. IV, f. $2-5$ (1861).

Nom. Jap. Tachi-mo.

Hab. in marshy places near Shoya. Fl. late Aug.-Sep.

Dist. Honshu, Yezo, Manchuria, Ussuri and Amur.

\title{
73. Hippuridaceae.
}

Hippuris L.

$371^{(1)}$ Hippuris vulgaris Linnaeus, Sp. Pl. ed. 1, I, p. 4 (1753).-Makino in Bot. Mag. Tokyo XVI, p. 215 (1902).-Kudo, Fl. Paramı. p. 136 (1922).Komarov et Klob.-Alisova, Key Pl. Far East. Reg. USSR. II, p. 780, t. 238, 5-6 (1932).-Tatewaki et Kobayasm, Contr. Fl. Aleut. p. 64 (19:34).

Nom. Jap. Sugina-mo.

Hab. in swamps near Shoya.

Dist. Honshu, Yezo, Kuriles, Saghalien, China, Manchuria, Amur, Ochotsk, Kamtchatka, Siberia, Dahuria, Altai, Caucasus, Europe, Greenland and N. \& S. America.

(To be continued.)

\section{Beobachtungen über Japanische Moosflora. XIb.}

Von

\section{Kyuichi Sakurai.}

Mit 5 Text-Figuren.

Eingegangen am 98. September, 1935.

Entodon (Erythropus) Osadae Sakurai sp. nov. (Fig. 6).

Corticola, robusta, caespitosa, caespitibus laxis, depressis, dilute flavoviridibus, opacis, mollibus. Caulis repens, ea. $3-5 \mathrm{~cm}$ longus, denudatus, ramosus, ramis erectis vel suberecitis, ca. $3 \mathrm{~cm}$ altis, cum foliis ca. $3 \mathrm{~mm}$ latis, dense complanate foliosis, simplicibus vel superne breve ramulosis. Folia sicca laxe adpressa, madida erecto-patentia, ramea haud decurrentia, e basi contracta oblonga apice breviore latiore subacuta vel obtusiuscula, dist- 


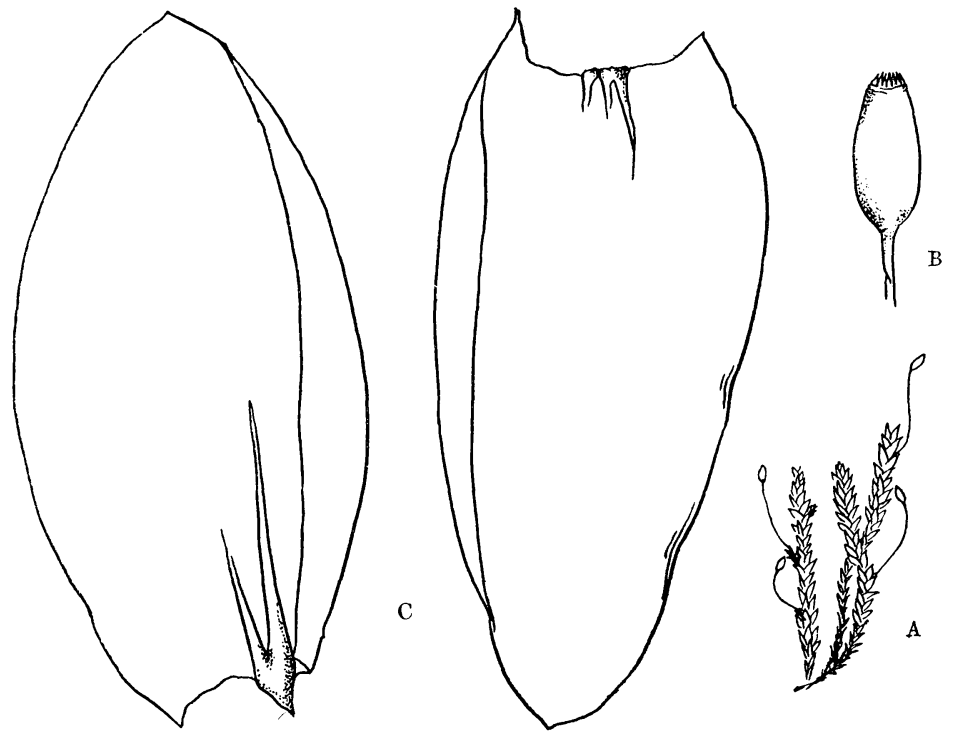

Fig. 6. Entodon Osadae SAK.

A. Fruchtende Pfl. $\times 1.5$. B. Kapsel. $\times 12.5$. C. Stengelbl. $\times 35$.

inctissime concava, marginibus erectis, integris, ca. $2,2 \mathrm{~mm}$ longa, $1 \mathrm{~mm}$ lata; nervis 2-4, plerumque binis, inaequalibus, indistinctis, sed $1 \mathrm{~mm}$ longis; cellulis anguste prosenchymatis, basin versus laxioribus, alaribus numerosis, quadratis, in toto vix chlorophyllosis. Bracteae perichaetii intt. longe lanceolatae sed vix piliforme attenuatae. Seta $3-4 \mathrm{~mm}$ longa, curvatula, levis. Theca erecta, ovalis, $1,1 \mathrm{~mm}$ longa, $0,6 \mathrm{~mm}$ crassa. Annulus fuscus, 2-4 cellulatus, persistus. Exostomii dentes lineari-lanceolati, obtusi, $0,24 \mathrm{~mm}$ longi, $0,03 \mathrm{~mm}$ lati, basi subfusci, apice subhyalini, in toto minutissime papillosi, ca. 20 lamellosi. Endostomium processus dentium fere brevior, carinatum, in carina haud perforatum, luteum, papillosum. Spori papillosi, 0,012 $\mathrm{mm}$ in diametro. Caetera desund.

Honshiu: Prov. Musashi, Horinouchi, (Leg. T. Osada Typus in Herb. K. Sakurai Nr. 458926 März 1931).

N.B. Habituell ist vorliegende Art E. compressus C. M. oder $E$. Challengeri PAR. ähnlich. Doch Statur ist doppelt breiter, trotzdem Seta weit kürzer.

Plagiothecium tokioense SaKurai sp. nov. (Fig. 7).

Terricola ; gracile, caespitosum, caespitibus laxiusculis, depressis, fuscoviridibus, haud nitidis. Caulis repens, per totam longitudinem, hic illic fusco-radiculosus, ca. $3-4 \mathrm{~cm}$ longus, laxe ramosus, ramis $1,0-1,2 \mathrm{~cm}$ longis, 
valde complanatis, cum foliis infra $2 \mathrm{~mm}$ latis, laxiuscule ramulosis, obtusis. Folia ramea patula, concaviuscula, haud decurrentia, asymmetrica, ovata, breviter acuminata, acuta, usque ad 1,0-1,3 mm longa, 0,5 mm lata, superne distincte serrulata, rarius inferne uno latere reflexi; nervis binis sed indistinctis; cellulis in medio folii $0,05-0,06 \mathrm{~mm}$ longis, $0,007-0,008 \mathrm{~mm}$ latis, alaribus haud numerosis, in toto levibus, pellucidis. Seta rubra, sicea flexuosula, $2 \mathrm{~cm}$ alta, levis. Theca conica, nutans, deoperuculata in siccitate sub ore constricta. Caetera desunt.

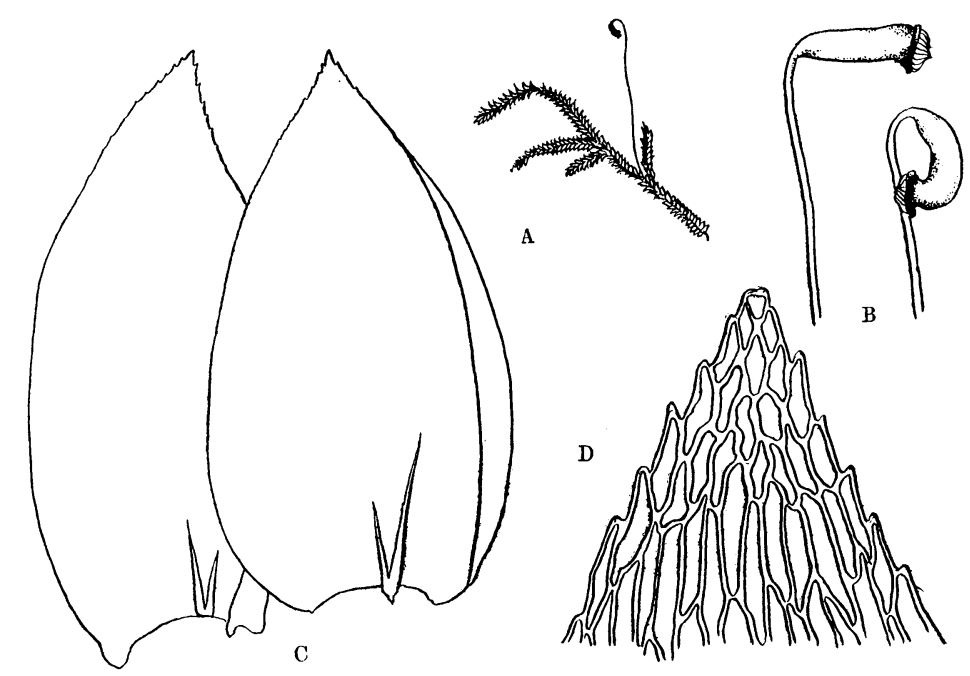

Fig. 7. Plagiothecium tolioense $\mathrm{SAK}$.

A. Fruchtende Pfl. $\times 1$. B. Kapsel. $\times 7.5$. C. Stengelb]. $\times$ 万0).

D. Blattspitze. vergr.

Honshiu: Tokio, auf schattigen Erdboden (Leg. T. Osada Typus in Herb.

K. Sakurai Nr. 455710 Mai 1932).

N.B. Vorliegende Art ist mit Pl.tosaense Brotrin. ïber seinen Blattbau sehr nahe verwandt, doch bei neuer Art sind Blätter 3-fach grösser, nur oben gezähnt und Álarzellen nicht deutlich.

var. vestitum SAKURAI var. nov. (Fig. 8).

Folia lateralia asymmetrica, subconcaviuscula, superne serrata. Propagulia numerosa, polymorpha, in axilis foliorum. Planta luteo-viridis, opaca. Honshiu: Prov. Musashi, Akabane, corticola (Leg. 'T. Osada Typus in Herb. K. Sakurai Nr. 5964 Mai 1931).

N.B. Blätter oberwärts nach einer Richtung gekrümmt, weit platter als die Hauptart. Brutkörper reichlich wie Figur zeigt. 

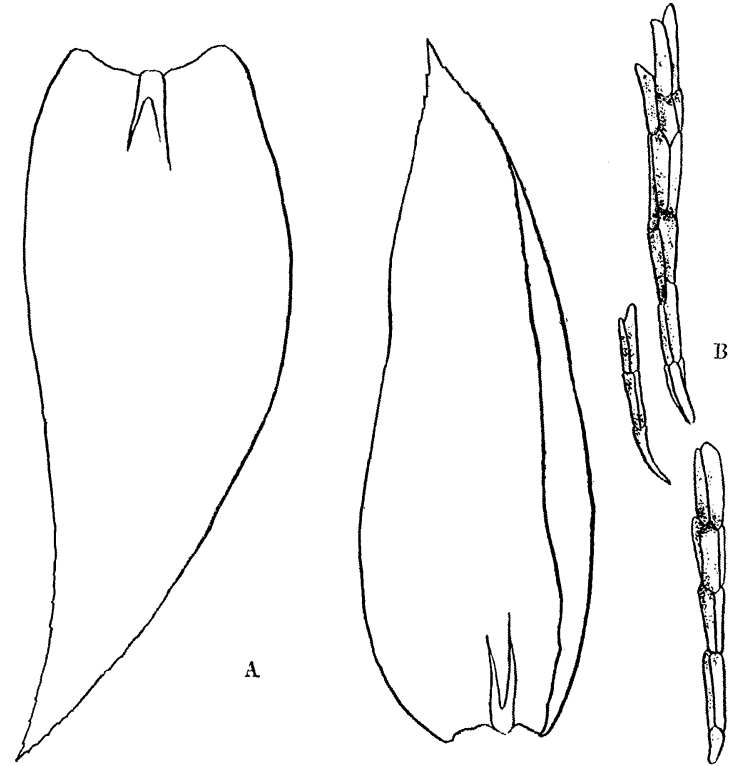

Fig. 8. Plagiothecium tokioense SAK. rar. vestitum $\mathrm{SAK}$. A. Stengelbl. $\times 60$. B. Brutkörper. vergr.

Plagiothecium Kanedae Sakurar sp. nov. (Fig. 9).

Planta robusta, caespitosa, caespitibus densissimis, superne laete viridibus, intus fuscescentibus, nitidis, mollibus, in toto valde depressis. Caulis repens, $3-5 \mathrm{~cm}$ longus, irregulariter ramosus, ramis ascendentibus, ca. $2 \mathrm{~cm}$ altis, valde complanatis. dense foliosis, obtusis. Folia lateralia paulum

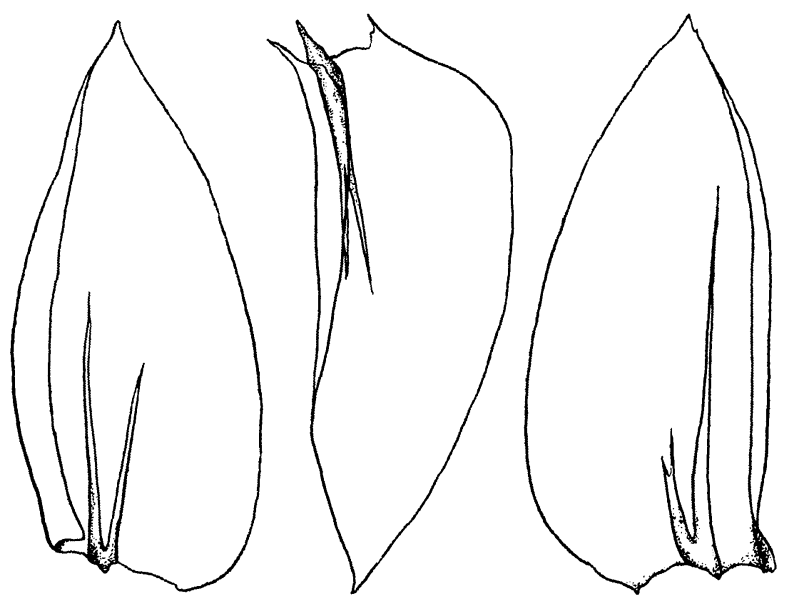

Fig. 9. Plagiothecium Kanedae SAk.

Stengelbl. $\times 20$ 
asymmetrica, concaviuscula, anguste decurrentia vel indistincta, e basi angusta late ovato-lanceolata, $3 \mathrm{~mm}$ longa, 1,5 $\mathrm{mm}$ lata, breve subacuta, marginibus uno latere incurvis, tantum superne indistincte serrulatis; costa valicla, polymorpha, plerumque bifurcata, rarius longe una vel trinervula, supra medium folii producta; cellulis anguste linearibus, levibus, basin versus laxioribus, alaribus et decurrentibus quadratis, pellucidis. Seta purpurea, $2 \mathrm{~cm}$ alta, flexuosula. Theca immaturata. Ad ligna putrida.

Kiushiu: Prov. Higo, Minamata, Mt. Yahazu (Leg. H. Kaneda Typus in

Herb. K. Sakurai Nr. 595925 März 1935); Taura-Dorf (Leg. H.

Kaneda in Herb. K. Sakurai Nr. 5047).

N.B. Anscheinend Sylvaticum-Gruppe. Man kann aber von sehr dicht geirängtem Habitus mit polymorpher Rippe sehr leicht bestimmen.

Plagiothecium pilosum Brotir. et YASUdA.

Kiushiu: Prov. Higo, Aso-gun, Shiromizu (Leg. N. Takaki in Herb. K. Sakurai Nr. 449617 Febr. 1935).

N.B. Bisher nur auf Formosa gesammelt.

Plagiothecium Yasudae Broth.

Kiushiu: Prov. Higo, Mt. Futaba (Leg. N. Takaki in Herb. K. SakuraI Nr. 389325 Juli 1934); Mt. Kitamuki (Leg. H. TAKahashi in Herb. K. SAKurai.

N.B. Bisher in Mittel-Honshiu selten gesammelt.

Oxyrhynchium rigidissimum Dix. et SAKURAI sp. nov. (Fig. 10).

Planta caespitosa, caespitibus valde densis, rigidissimis, superne viridibus, inferne fuscescentibus. Caulis repens, ca. $5 \mathrm{~cm}$ longus, plerumque denudatus, irregulariter dense ramosus, subpinnam ramulosus. Folia ramea sicca laxe imbricata, madida erecto-patentia, obovata vel ovalia, subobtusa vel subacuta, $0,8-1,0 \mathrm{~mm}$ longa, $0,5 \mathrm{~mm}$ lata, in toto vel supra $2 / 3$ minute serrata; nervo lato ad $4 / 5$ folii evanido, apice indistincte exstante; cellulis prosenchymatis, levibus, basin versus laxioribus, alaribus haud vesiculosis. Seta ca. 1,2-1,4 cm alta, levis. Theca suberceta, oblongo-cylindrica, parce curvatula, collo distincto, $1,8 \mathrm{~mm}$ longa, $0,8 \mathrm{~mm}$ crassa. Peristomium duplex. Exostomii dentes lanceolato-subulati, superne hyalini, papillosi, $0,35 \mathrm{~mm}$ longi, basi connati, linea media flexuosula, strato dorsali transversim striatulo, strato ventrali densissime lamelloso; endostomium luteum, minutissime papillosum, corona basilaris alta, processus dentium fere longitudinis, carinatus, in carina rimosulis; cilia bina, haud bene evoluta. Spori virides, papillosi, 0,015 $\mathrm{mm}$ in diametro. Operculum longe rostratum. Kiushiu: Prov. Higo, Kawara-Dorf (Leg. N. Takaki Typus in Herb. K.

SAKURAi Nr. 486623 Sept. 1934). Auf Felsen im Bächlein. 


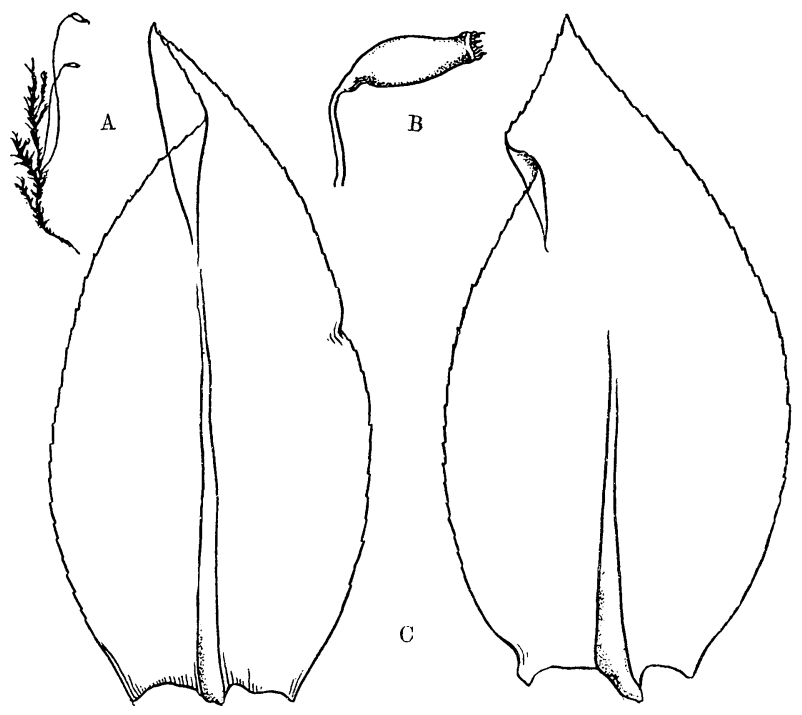

Fig. 10. Oxyrhynchium rigidissimum Dix. et SAK.

A. Fruchtende Pfl. im trocknen Zustande. $\times 1.5$. B. Kapsel. $\times$. 7.5. C. Stengelb1. $\times 70$.

N.B. Von sehr rigidem drahtartigem Habitus kam man sofort bestimmen.

Pseudoleskea papillarioides C. MI.

Honshiu: Prov. Shinano, Mt. Yarigatake, in hochalpiner Lage (Leg. T. Osada in Herb. K. Sakurai Nr. 37552 Aug. 1934, det. I. Thériot').

N.B. Neu für japanische Flora (auch in Korea ?).

(Fortsetzung folgt.)

\section{Notes on Japanese Desmids, with Special Reference to the Newly Found Species. III.}

By

\section{Yoshikazu Okada.}

With 1 Plate.

Receiver November 15, 1935.

25) Xanthidium cristatum BRÉB. forma depressa HoMfeld.

(Pl. IV, figs. 1, 1').

Homfeld, Desm. Nordwestdeutch. 1929, p. 65, pl. 8, fig. 85. 